\title{
Effect of Autologous Serum Eye Drops in Patients with Sjögren Syndrome-related Dry Eye: Clinical and In Vivo Confocal Microscopy Evaluation of the Ocular Surface
}

\author{
FRANCESCO SEMERARO ${ }^{1}$, ELIANA FORBICE ${ }^{1}$, GIUSEPPE NASCIMBENI $^{1}$, MARCO TAGLIETTI $^{2}$, \\ VITO ROMANO ${ }^{3}$, GERMANO GUERRA ${ }^{4}$ and CIRO COSTAGLIOLA ${ }^{5,6}$ \\ ${ }^{1}$ Opthamology Clinic, Depatment of Medical and Surgical Specialties, \\ Radiological Specialties and Public Health, University of Brescia, Brescia, Italy; \\ ${ }^{2}$ Rheumatology and Clinical Immunology Unit, University of Brescia, Brescia, Italy; \\ ${ }^{3}$ Department of Corneal and External Eye Diseases, St. Paul's Eye Unit, \\ Royal Liverpool University Hospital, Liverpool, U.K.; \\ ${ }^{4}$ Anatomy Institute, ${ }^{5}$ Eye Clinic, Department of Medicine \& Health Sciences, \\ University of Molise, Campobasso, Italy; \\ ${ }^{6}$ IRCCS Neuromed, Pozzilli, Isernia, Italy
}

\begin{abstract}
Aim: To evaluate in vivo changes after therapy using autologous serum (AS) eye drops in Sjögren's syndrome (SS)-related dry eyes by confocal microscopy. Patients and Methods: In this study, 24 patients with SSrelated dry eyes [12 in AS eye drop therapy and 12 in artificial tear (AT) therapy] and 24 healthy volunteers were recruited. Ocular Surface Disease Index (OSDI), central corneal thickness, tear film, break-up time, corneal and conjunctival staining, Schirmer's test and corneal confocal microscopy were investigated. Results: Tear production, tear stability, corneal staining, inflammation, and central corneal thickness, Langherans cells, activated keratocytes, intermediate epithelial cell density, nerve tortuosity, number of sub-basal nerve branches, and number of bead-like formations differed between patients and controls $(p<0.0001)$. The AT and AS groups differed in the OSDI, number of branches, and number of beadings $(p<0.0001)$. Conclusion: AS eye drops improve symptoms and confocal microscopy findings in SS-related dry eyes.
\end{abstract}

Aqueous-deficient dry eye (DE) may be subdivided into Sjögren's syndrome-associated dry eye (SSDE), and nonSjögren's syndrome-associated dry eye (NSSDE), that is

Correspondence to: Vito Romano, MD, St. Paul's Eye Unit, Royal Liverpool University Hospital, 8Z Link, Prescot Street, Liverpool L7 8XP, U.K. Tel: +44 1517062134, Fax: +44 01517065861, email: vito.romano@gmail.com

Key Words: Autologous serum eye drops, Sjögren syndrome, dry eye, confocal microscopy. mainly age-related (1). Although not as common as agerelated DE, SSDE is considered the prototype for dry eye disease due to its severity (2). Sjögren's syndrome is a chronic inflammatory autoimmune disease which affects the lacrimal and salivary glands. Confocal microscopy is increasingly used in in vivo studies of morphological changes of the cornea and conjunctiva in a wide range of physiological and pathological states $(3,4)$. It has also been used in several studies to investigate alterations in the subepithelial plexus, epithelium, and stroma of the corneas of patients suffering from DE disease $(2,4-7)$. In vivo confocal microscopy (IVCM) has proven to be capable of identifying different corneal structural aspects, which are considered pathognomonic of $\mathrm{DE}$ disease and may be useful in monitoring the disease and the effectiveness of therapy. IVCM has allowed the study of the nerve plexus, where it highlighted the presence of corneal neuropathy and was also able to highlight, in corneas of patients with DE disease, other fundamental parameters indicative of corneal pathology, such as a reduced number of epithelial cells, increase of Langerhans cells and activated keratocytes (2, 5-7).

The use of autologous serum (AS) for treating ocular surface disorders began in 1970 to treat ocular alkali burns (8). To date, it has been used for the treatment of different diseases of the ocular surface, such as persistent epithelial defects (9-15), DE (16-23), neurotropic keratopathy (21), recurrent erosion syndrome $(22,23)$, superior limbic keratoconjunctivitis (24), and chemical injuries $(25,26)$. Human serum presents strong similarity to natural tears and contains many important components including: i) epithelial growth factor, which accelerates epithelial cell migration and has anti-apoptotic effects (27); ii) transforming growth factor- $\beta$ 
(TGF $\beta$ ), involved in the epithelial and stromal repair process (28); iii) vitamin A, which may prevent epithelial squamous metaplasia (29) and modulate the expression of thrombospondin 1 (30); iv) thrombospondin 2, vascular endothelial growth factor A, and metallopeptidase 9, which together with TGF $\beta$ promote wound healing (31); v) albumin, which has anti-apoptotic activity (32); and vi) $\alpha-2$ macroglobulin, which exhibits anti-collagenase activity; and fibronectin, which is important in cell migration $(4,33)$.

Additionally, AS also contains neuronal factors such as substance $\mathrm{P}$ and insulin-like growth factor 1 , which may have a role in corneal epithelial migration and adhesion (34). Moreover, AS contains immunoglobulins (Ig) such as IgG and $\operatorname{IgA}$, and lysozyme, which provide bactericidal and bacteriostatic effects (35). It is also free of preservatives (36) which could potentially induce toxic or allergic reactions (37), and its osmolality and biomechanical properties are similar to those of natural tears.

In a previous study, we recorded a significant improvement in the signs and symptoms after therapy with eye drops in patients with different ocular surface disorders (25). The main objective of this study was to investigate the mechanisms by which AS eye drops are able to ameliorate alterations in the corneal epithelium and sub-basal nervous plexus in patients with SSDE.

\section{Patients and Methods}

Patients. The study was conducted from January 2013 to July 2014. Twenty-four patients with SSDE were recruited from the Cornea Unit of Spedali Civil Hospital (Brescia, Italy). The patients were divided into two groups according to the assigned treatment: 12 patients were treated with AS eye drops (AS group) (all women; mean age $=54.00 \pm 9.88$ years $)$; and 12 patients with artificial tears (AT group) (all women; mean age $=54.67 \pm 16.16$ years). The division between the two groups was made in a randomized order assigning 12 patients observed before January 2013 to the AT group and the 12 patients from January to July 2013 to the AS group.

Twenty-four age-matched women were also recruited as controls (control group; all women; mean age $=45.17 \pm 12.95$ years).

Written informed consent was obtained from all participants after a detailed explanation of the procedure used and of the aim of this work. The Institutional Review Board of the Spedali Civil Hospital (Brescia, Italy) Ethics Committee approved the study (15A09709). The Declaration of Helsinki was followed. All patients had a screening visit. The following exclusion criteria were used: age under 18 years; inability to complete the questionnaire or understand the procedures; use of contact lenses; history of ocular trauma or surgery, drug allergy, systemic or ocular disease (except Sjögren's syndrome for AS and AT groups); use of topical medications that may affect the corneal and ocular surfaces (except the use of AS or AT in respective groups).

All patients with SS were receiving therapy with hydroxychloroquine (200 mg, once a day).

The diagnosis of primary and secondary Sjögren's syndrome were made by a specialized rheumatologist in accordance with the criteria proposed by the American-European Consensus Group (26).
The diagnosis of DE disease was made on the basis of Schirmer's test 1 results measuring less than $5 \mathrm{~mm}$ with/without tear film break-up time (TBUT) of less than $10 \mathrm{sec}$, accompanied by complaints of ocular diseases. None of the controls had any complaints of ocular surface irritation, nor did they demonstrate any anterior segment abnormalities on biomicroscopic examination.

The AS group was treated with AS eye drops five times a day for the entire follow-up period (1 year). The AT group was treated with AT eye drops five times a day for the entire follow-up period. No topical treatment was performed for the control group.

AS drops were produced by our laboratory according to a standardized and validated method (25).

Clinical evaluation. The Ocular Surface Disease Index (OSDI) questionnaire was used to grade the symptoms of DE in participants (38). All participants underwent a complete examination of the ocular surface of both eyes, which included Schirmer's test, TBUT, corneal and conjunctival staining, corneal thickness, and IVCM analysis of the central cornea.

Schirmer's test was performed without anesthesia for 5 minutes with the patient's eyes closed. TBUT was measured by instilling fluorescein into the inferior fornix and calculating the average of two consecutive breakup times. Corneal and conjunctival staining were evaluated using the Oxford scale after instillation of fluorescein under a yellow filter, and the Van Bijsterveld score was evaluated after instillation of lissamine green and white light. Corneal thickness measurements were performed using Pentacam (Oculus GmbH, Wetzlar, Germany).

A minimum of 10 images of the epithelium and anterior stroma were acquired sequentially, and the five images best in focus were analyzed. Images of the sub-basal nervous plexus of the central cornea were acquired by focusing the microscope beneath the basal epithelium. Approximately 10 images of the corneal sub-basal nervous plexus were acquired in the central cornea, and the five images demonstrating the most sub-basal nerve fibers were selected for quantitative analysis. Images of corneal sub-basal nerves were analyzed retrospectively using NeuronJ (Biomedical Imaging Group, Erasmus University Medical Center, Rotterdam, the Netherlands) by a single researcher. The images were acquired in two sizes and with a definition of $384 \times 384$ pixels, equivalent to an area of $400 \times 400 \mu \mathrm{m}$.

The different parameters measured using confocal microscopy were as follows: Sub-basal corneal nerves $(26,27)$. Density of corneal nerve: This refers to the total length of nerve fibers visible within a frame, expressed in $\mathrm{mm} / \mathrm{mm}^{2}$. Number of sub-basal nerves: This refers to the total number of parallel nerves visible in one frame, expressed in number of fibers $/ \mathrm{mm}^{2}$. Number of sub-basal nerve branches: This refers to the sum of the number of nerve branches seen in one frame, and was manually calculated after elaboration with Neuronj (Biomedical Imaging Group), and expressed as the number of branches $/ \mathrm{mm}^{2}$. Number of bead-like formations: This is defined as the number of such formations along $100 \mu \mathrm{m}$ of fiber. Beads are highly reflective broadening portions of the sub-basal nerve. Tortuosity of nerves: Classified into four grades according to a previously validated scale (27).

Epithelial cell density. This was evaluated at the level of the basal epithelial and intermediate layers. In all cases, cell density was determined manually, using a cell counting procedure present in the Neuronj software (Biomedical Imaging Group), taking into consideration the whole area marked as being available for the cell 
count. The cells partially contained in the area analyzed were counted only along the right and lower margins. The results are expressed as the number of cells $/ \mathrm{mm}^{2}$.

Langerhans cells. This refers to bright, corpuscular, or spicular elements that appear to be scattered among nerve fibers or within the corneal epithelium at the basal level, or in the sub-basal nerve plexus. The cell density was manually determined, and the results expressed as the number of cells/frame.

Activated keratocytes. This refers to hyper-reflective stromal cells with an elongated and dendritic appearance; the cell density was manually determined, and the results expressed as the number of cells $/ \mathrm{mm}^{2}$ (24).

Statistical analysis. All the data are expressed as mean \pm SD values Comparisons between the control and the Sjogren groups and between the AS and AT groups were made using the $t$-test.

Linear regression analysis was performed to test statistical associations between DE tests and patient's symptoms; DE tests and quantitative confocal microscopic variables; and within confocal microscope variables $(p<0.01)$.

\section{Results}

Clinical data. The demographic and clinical data and the comparisons between the SS and control groups are shown in Table I. No significant differences were present in the demographic data between the two groups.

All the patients with SS had significantly more symptoms compared to the control group, as assessed by the OSDI $(p<0.0001)$.

Tear production was significantly lower in patients of the AS and AT groups than in controls $(p<0.0001)$. Additionally, tear stability was significantly lower in these patients than in the controls $(p<0.0001)$. Corneal fluorescein and lissamine staining was demonstrated as severe in patients with SS, but absent from the control group $(p<0.0001)$. Moderate and severe inflammation was present in all patients with SS with significant differences between the SS group and the control group ( $p=0.001)$. Central corneal thickness was significantly less in the patients with SS when compared to the control group $(p<0.0001)$.

The comparison of demographic and clinical data between the AS and AT groups is shown in Table II. No significant differences were present in demographic data between the AS and AT groups. The OSDI data significantly differed, with fewer symptoms in the AS group $(p=0.0027)$. None of the clinical data were statistically significant: there were no differences among tear production $(p=0.8)$, tear stability $(p=0.025)$, corneal fluorescein and lissamine staining (fluorescein, $p=0.05$ ) (lissamine, $p=0.07$ ), conjunctival inflammation $(p=0.14)$, and corneal thickness $(p=0.076)$.

Confocal microscopy. Table III shows the results of the confocal microscopy data for the SS and control groups.
Table I. Demographic and clinical test results in DE disease and control groups.

\begin{tabular}{lccc}
\hline Parameters & DE group & Control group $p$-Value \\
\hline Patients, $\mathrm{n}$ & 24 & 24 & \\
Gender, female & 24 & 24 & \\
Age, years & $54.31 \pm 11.49$ & $48.88 \pm 6.50$ & 0.05 \\
Disease duration, years & $13.31 \pm 5.75$ & - & \\
OSDI, AU & $19.46 \pm 7.02$ & $1.00 \pm 0.59$ & $<0.0001$ \\
Schirmer test, AU & $1.69 \pm 0.47$ & $0.00 \pm 0.00$ & $<0.0001$ \\
TBUT, s & $7.85 \pm 1.64$ & $12.67 \pm 1.63$ & $<0.0001$ \\
Oxford scale, AU & $1.08 \pm 1.02$ & $0.00 \pm 0.00$ & $<0.0001$ \\
Van Bijsterveld, AU & $0.92 \pm 0.63$ & $0.00 \pm 0.00$ & $<0.0001$ \\
Conjunctival inflammation, AU & $1.46 \pm 0.65$ & $1.00 \pm 0.00$ & 0.001 \\
Corneal thickness, $\mu \mathrm{m}$ & $549.08 \pm 29.51$ & $593.83 \pm 8.45$ & $<0.0001$ \\
& & &
\end{tabular}

AU: Arbitrary units; OSDI: Ocular Surface Disease Index; TBUT: tear film break-up time.

Table II. Demographic and clinical test results in artificial tear (AT) and autologous serum $(A S)$ groups.

\begin{tabular}{lccc}
\hline Parameter & AT group & AS group & $p$-Value \\
\hline Patients, n & 12 & 12 & \\
Gender, female & 12 & 12 & \\
Age, years & $54.00 \pm 7.27$ & $54.67 \pm 15.41$ & 0.89 \\
Disease duration, years & $12.29 \pm 6.37$ & $14.50 \pm 4.93$ & 0.34 \\
OSDI, AU & $23.29 \pm 5.14$ & $15.00 \pm 7.46$ & 0.0027 \\
Schirmer test, AU & $1.71 \pm 0.47$ & $1.67 \pm 0.49$ & 0.8 \\
TBUT, s & $7.00 \pm 1.47$ & $8.83 \pm 1.27$ & 0.025 \\
Oxford scale, AU & $0.71 \pm 0.91$ & $1.51 \pm 1.00$ & 0.05 \\
Van Bijsterveld, AU & $0.71 \pm 0.73$ & $1.17 \pm 0.39$ & 0.07 \\
Conjunctival inflammation, AU & $1.29 \pm 0.47$ & $1.67 \pm 0.78$ & 0.14 \\
Corneal thickness, $\mu \mathrm{m}$ & $558.57 \pm 35.81$ & $538.00 \pm 14.67$ & 0.076 \\
\hline
\end{tabular}

AU: Arbitrary units; OSDI: Ocular Surface Disease Index; TBUT: tear film break-up time.

Statistically significant differences were found in tortuosity of nerves $(p<0.0001)$, number of sub-basal nerve branches $(p<0.0001)$, and number of bead-like formations $(p<0.0001)$ in the comparison between patients and the controls. In relation to the number and density of sub-basal nerves, no significant differences were observed between patients with $\mathrm{DE}$ disease and the control group (number, $p=0.2$ ) (density, $p=0.51$ )

Intermediate epithelial cell density was significantly low in patients with DE disease than in the control group $(p<0.0001)$. The basal epithelial cell density shows nonstatistically significant differences between the two groups $(p=0.17)$. Langerhans cell and activated keratocytes were found at a significantly lower frequency in patients with DE disease than in the control group (Langerhans, $p<0.0001$; keratocytes, $p<0.0001)$. 
Table III. Findings from in vivo confocal microscopy in dry eye (DE) disease and control groups.

\begin{tabular}{lccc}
\hline Parameter & DE group & Control group & $p$-Value \\
\hline Patients, $\mathrm{n}$ & 24 & 24 & 0.51 \\
Density of sub-basal nerves, $\mathrm{mm} / \mathrm{mm}^{2}$ & $11.44 \pm 5.14$ & $10.7 \pm 2.06$ & 0.2 \\
Sub-basal nerves, $\mathrm{n} / \mathrm{mm}^{2}$ & $31.17 \pm 9.51$ & $28.13 \pm 6.9$ & $<.0001$ \\
Branchings, $\mathrm{n} / \mathrm{mm}^{2}$ & $27.82 \pm 6.61$ & $17.6 \pm 8.53$ & $<.0001$ \\
Beadings, $\mathrm{n} / 100 \mu \mathrm{m}$ of nerve length & $19.23 \pm 2.69$ & $1.5 \pm 0.51$ & $<0.0001$ \\
Tortuosity of nerves, AU & $2.62 \pm 0.64$ & $6240.00 \pm 620.80$ & 0.17 \\
Density of basal epithelium, cells $/ \mathrm{mm}^{2}$ & $6440.62 \pm 560.11$ & $<658.50 \pm 434.84$ & $<0.0001$ \\
Density of intermediate epithelium, cells $/ \mathrm{mm}^{2}$ & $4637.15 \pm 555.34$ & $0.00 \pm 0.00$ & $<0.0001$ \\
Activated Langerhans cells, $\mathrm{n} / \mathrm{frame}$ & $3.92 \pm 2.81$ & $35.83 \pm 2.46$ & $<0.0001$ \\
Activated keratocytes, $\mathrm{n} / \mathrm{mm} 2$ & $46.62 \pm 3.41$ & &
\end{tabular}

AU: Arbitrary units.

Table IV. Findings from in vivo confocal microscopy in artificial tear (AT) and autologous serum (AS) groups.

\begin{tabular}{lccc}
\hline Parameter & AT group & AS group & $p$-Value \\
\hline Patients, $\mathrm{n}$ & 12 & 12 & 0.06 \\
Density of sub-basal nerves, $\mathrm{mm} / \mathrm{mm}^{2}$ & $13.20 \pm 6.35$ & $9.39 \pm 1.95$ & 0.64 \\
Sub-basal nerves, $\mathrm{n} / \mathrm{mm}^{2}$ & $31.99 \pm 12.69$ & $30.21 \pm 3.61$ & $<0.0001$ \\
Branchings, $\mathrm{n} / \mathrm{mm}^{2}$ & $32.57 \pm 5.50$ & $17.28 \pm 1.22$ & $<.0001$ \\
Beadings, $\mathrm{n} / \mathrm{100} \mu \mathrm{m}$ of nerve length & $21.00 \pm 2.08$ & $2.50 \pm 0.52$ & 0.4 \\
Tortuosity of nerves, AU & $2.71 \pm 0.73$ & $6844.00 \pm 48.87$ & 0.03 \\
Density of basal epithelium, cells/mm ${ }^{2}$ & $6094.86 \pm 567.76$ & $4585.83 \pm 354.31$ & 0.87 \\
Density of intermediate epithelium, cells $/ \mathrm{mm}^{2}$ & $4681.14 \pm 694.51$ & $3.50 \pm 3.12$ & 49 \\
Activated Langerhans cells, $\mathrm{n} / \mathrm{frame}$ & $4.29 \pm 2.58$ & $46.17 \pm 2.04$ & 0.54 \\
Activated keratocytes, $\mathrm{n} / \mathrm{mm} 2$ & $47.00 \pm 4.30$ & & \\
\hline
\end{tabular}

AU: Arbitrary units.

The results and the comparison between the AS and AT groups are shown in Table IV. There were no differences between the confocal data of the AT and AS groups, with exception of the number of branches $(p<0.0001)$ and the number of beadings $(p<0.0001)$, which were lower in the AS group.

Correlation. As significant correlation was found between patients' symptoms and standard dry eye test such as TBUT, Schirmer's test. The dry eye test results also correlated with each other: TBUT vs. Schirmer's test, TBUT vs. lissamine, fluorescein $v s$. lissamine. Central corneal thickness showed significant correlation with the Schirmer's test, fluorescein staining, and lissamine staining (Tables V and VI).

Confocal data correlated with clinical data; the patients' symptoms in the OSDI test correlated with the number of branches, the number of beadings, the tortuosity of nerves, the intermediate epithelial cell density, and the number of activated keratocytes. TBUT correlated with the number of beadings, the tortuosity of nerves, the intermediate epithelial cell density, and the number of activated keratocytes.
Schirmer's test results correlated with the number of beadings, the tortuosity of nerves, the intermediate epithelial cell density, the number of activated keratocytes, and the number of activated Langerhans cells. Lissamine staining correlated with the density of the intermediate epithelial cells and the number of activated keratocytes.

Confocal data also correlated with each other; the number of beadings correlated with the number of branches and the tortuosity of nerves. The number of activated keratocytes correlated with the number of activated Langerhans cells.

\section{Discussion}

In a previous study, we found that patients with DE disease of different etiologies showed improvement in both clinical signs and symptoms after therapy with AS eye drops (24).

In the present study, our objective was to demonstrate the changes occurring in the ocular surface after use of AS eye drops. For this, we supplemented the usual clinical tests with the IVCM study of the cornea; in fact, it has been demonstrated that traditional clinical diagnostic methods are 
Table V. Clinical correlations.

\begin{tabular}{|c|c|c|c|c|c|c|}
\hline & $\begin{array}{c}\text { Corneal thickness, } \\
\mu \mathrm{m}\end{array}$ & $\begin{array}{l}\text { Schirmer test, } \\
\text { AU }\end{array}$ & $\begin{array}{l}\text { TBUT, } \\
\text { s }\end{array}$ & $\begin{array}{l}\text { Van Bijsterveld } \\
\text { scale, } \mathrm{AU}\end{array}$ & $\begin{array}{c}\text { Oxford } \\
\text { scale, } \mathrm{AU}\end{array}$ & $\begin{array}{c}\text { Conjunctival } \\
\text { inflammation, } \mathrm{AU}\end{array}$ \\
\hline OSDI, arbitrary units & $p>0.01$ & $\begin{array}{c}p<0.0001 \\
\mathrm{r}^{2}=0.6\end{array}$ & $\begin{array}{c}p<0.0001 \\
\mathrm{r}^{2}=0.65\end{array}$ & $p>0.01$ & $p>0.01$ & $p>0.01$ \\
\hline Conjunctival inflammation, $\mathrm{AU}$ & $p>0.01$ & $p>0.01$ & $p>0.01$ & $p>0.01$ & $p>0.01$ & \\
\hline Oxford scale, $\mathrm{AU}$ & $\begin{array}{l}p=0.01 \\
\mathrm{r}^{2}=0.32\end{array}$ & $p>0.01$ & $p>0.01$ & $\begin{array}{c}p<0.0001 \\
\mathrm{r}^{2}=0.63\end{array}$ & & \\
\hline Van Bijsterveld scale, AU & $\begin{array}{c}p=0.0042 \\
\mathrm{r}^{2}=0.39\end{array}$ & $p>0.01$ & $\begin{array}{c}p=0.0084 \\
\mathrm{r}^{2}=0.34\end{array}$ & & & \\
\hline TBUT, s & $p>0.01$ & $\begin{array}{c}p=0.0003 \\
\mathrm{r}^{2}=0.55\end{array}$ & & & & \\
\hline Schirmer test, AU & $\begin{array}{l}p=0.01 \\
\mathrm{r}^{2}=0.32\end{array}$ & & & & & \\
\hline
\end{tabular}

AU: Arbitrary units; OSDI: Ocular Surface Disease Index; TBUT: tear film break-up time.

Table VI. Correlations of findings of in vivo confocal microscopy with clinical tests.

\begin{tabular}{|c|c|c|c|c|c|c|c|}
\hline & $\begin{array}{l}\text { OSDI, } \\
\text { AU }\end{array}$ & $\begin{array}{c}\text { Conjunctival } \\
\text { inflammation, } \mathrm{AU}\end{array}$ & $\begin{array}{c}\text { Oxford scale, } \\
\text { AU }\end{array}$ & $\begin{array}{l}\text { Van Bijsterveld } \\
\text { scale, } \mathrm{AU}\end{array}$ & $\begin{array}{c}\text { TBUT, } \\
\mathrm{s}\end{array}$ & $\begin{array}{l}\text { Schirmer test, } \\
\text { AU }\end{array}$ & $\begin{array}{c}\text { Corneal thickness, } \\
\mu \mathrm{m}\end{array}$ \\
\hline Density of sub-basal nerves, $\mathrm{mm} / \mathrm{mm}^{2}$ & $p>0.01$ & $p>0.01$ & $p>0.01$ & $p>0.01$ & $p>0.01$ & $p>0.01$ & $p>0.01$ \\
\hline Sub-basal nerves, $\mathrm{n} / \mathrm{mm}^{2}$ & $p>0.01$ & $p>0.01$ & $p>0.01$ & $p>0.01$ & $p>0.01$ & $p>0.01$ & $p>0.01$ \\
\hline Branchings, $\mathrm{n} / \mathrm{mm}^{2}$ & $\begin{array}{c}p=0.0092 \\
r^{2}=0.35\end{array}$ & $p>0.01$ & $p>0.01$ & $p>0.01$ & $p>0.01$ & $p>0.01$ & $p>0.01$ \\
\hline Beadings, $\mathrm{n} / 100 \mu \mathrm{m}$ of nerve length & $\begin{array}{c}p=0.0003 \\
\mathrm{r}^{2}=0.55\end{array}$ & $p>0.01$ & $p>0.01$ & $p>0.01$ & $\begin{array}{c}p=0.0009 \\
\mathrm{r}^{2}=0.49\end{array}$ & $\begin{array}{l}p=0.01 \\
\mathrm{r}^{2}=0.31\end{array}$ & $p>0.01$ \\
\hline Tortuosity of nerves, AU & $\begin{array}{c}p=0.010 \\
\mathrm{r}^{2}=0.31\end{array}$ & $p>0.01$ & $p>0.01$ & $p>0.01$ & $\begin{array}{l}p=0.01 \\
\mathrm{r}^{2}=0.28\end{array}$ & $\begin{array}{l}p=0.01 \\
\mathrm{r}^{2}=0.35\end{array}$ & $p>0.01$ \\
\hline Density of basal epithelium, cell $/ \mathrm{mm}^{2}$ & $p>0.01$ & $p>0.01$ & $p>0.01$ & $p>0.01$ & $p>0.01$ & $p>0.01$ & $p>0.01$ \\
\hline $\begin{array}{l}\text { Density of intermediate } \\
\text { epithelium, cells } / \mathrm{mm}^{2}\end{array}$ & $\begin{array}{c}p=0.0017 \\
\mathrm{r}^{2}=0.45\end{array}$ & $p>0.01$ & $p>0.01$ & $\begin{array}{c}p=0.0013 \\
\mathrm{r}^{2}=0.46\end{array}$ & $\begin{array}{c}p=0.0008 \\
r^{2}=0.49\end{array}$ & $\begin{array}{c}p=0.005 \\
\mathrm{r}^{2}=0.38\end{array}$ & $p>0.01$ \\
\hline Activated keratocytes, $\mathrm{n} / \mathrm{mm}^{2}$ & $\begin{array}{c}p=0.0001 \\
\mathrm{r}^{2}=0.49\end{array}$ & $p>0.01$ & $p>0.01$ & $\begin{array}{c}p=0.0042 \\
r^{2}=0.39\end{array}$ & $\begin{array}{c}\mathrm{p}<0.0001 \\
\mathrm{r}^{2}=0.67\end{array}$ & $\begin{array}{c}p=0.0007 \\
\mathrm{r}^{2}=0.5\end{array}$ & $p>0.01$ \\
\hline Activated Langerhans cells, $\mathrm{n} /$ frame & $p>0.01$ & $p>0.01$ & $p>0.01$ & $p>0.01$ & $p>0.01$ & $\begin{array}{c}p=0.0076 \\
r^{2}=0.35\end{array}$ & $p>0.01$ \\
\hline
\end{tabular}

AU: Arbitrary units; OSDI: Ocular Surface Disease Index; TBUT: tear film break-up time.

unsatisfactory and limited in the evaluation of DE disease. The use of IVCM introduces a new approach to the study of corneal morphology, offering resolutions comparable to those obtained in histological examinations, but in a noninvasive, safe and reproducible way $(26,27)$.

We analyzed the symptoms, clinical signs, and confocal aspects of patients receiving treatment with AS eye drops, compared to those being treated with AT. The data from these two groups were compared to data of healthy controls. In order to acquire more consistent and unequivocal data, we decided to evaluate only patients with a single etiology of DE, i.e. SSDE, which is considered the prototype of DE.
Our study shows that the symptoms reported using OSDI are closely related with the severity of the clinical signs in patients suffering from SS; similar to our findings, one previous study found a surprising association between the OSDI evaluation and some clinical signs in patients with SS (6). However, previous literature does not show a unique relationship between these aspects and, most articles emphasize a scant association between the signs and symptoms in DE disease $(28-30,37)$, while few others report occasional associations $(2,31)$ or inverse correlations (32). Such heterogeneous data are probably due to the small sample size used in many past studies, and because of the characteristics of the different questionnaires used. 
We also analyzed the corneal changes using IVCM. Corneal sub-basal nerves in patients with SDDE showed greater tortuosity, increased bead-like formations, and increased number of beadings. Such patients also exhibited a reduced number of epithelial cells, an increase in Langerhans cells and activated keratocytes, and a reduced corneal thickness. However, there is not agreement regarding IVCM findings in the literature. When evaluating sub-basal nerve density, some authors observed a decrease (2-10, 33 , $34)$, while others found no change $(35,36)$, or, in accordance with our study, an increase in density (37). However, most of these studies were conducted on groups with unequal numbers of patients with SSDE and NSSDE, making the comparison between studies difficult. We have also found that the structural and functional alterations of the sub-basal corneal nerve were related to the severity of DE disease.

The tortuosity and the number of beads seemed to correlate with the data from the OSDI, TBUT, and Schirmer's tests, but not with the results of vital staining, or with visible inflammation. In addition, the density of the epithelium correlated with OSDI, TBUT, Schirmer's test, but also to lissamine staining. On the other hand, corneal thickness correlated with the results of vital staining with both lissamine and fluorescein. These associations are important because they highlight the fact that the symptoms correlate with lacrimal function and nervous morphology, and poorly with vital staining and conjunctival inflammation.

Significant correlations were also recorded between fiber tortuosity, superficial epithelial density and anterior activated keratocytes density (6). These correlations make sense because all of this data are deemed as an index of high metabolic activity, possibly directed to repair the alterations observed at the epithelial level (39). Benitez del Castillo et al. highlighted the key role played by keratocytes in the inflammatory process linked with DE disease (2). As a matter of fact, the activated keratocytes produce nervous growth factors that are a contribution to the processes of activation and reorganization of the nerve fibers of the subbasal plexus (37). A past study revealed a significantly higher number of hyper-reflective stromal cells, believed to be keratocytes, in a specific stage of metabolic activation in patients with DE disease (5).

In addition to the number of activated keratocytes, the number of bead-like formations can also be interpreted as a confocal sign of ocular surface disease activity (7). The bead-like formations are characteristic of metabolically active nerve fibers containing transmitters, and the increased number found in DE disease cases indicates an attempt to improve the abnormal epithelial tropism $(34,36,37,40)$. The chronic inflammation and diminished volume and clearance of tears enriched with pro-inflammatory cytokines such as interleukin (IL)-1 and IL6 lead to the activation of keratocytes, which synthesize nerve growth factor and other factors of nerve growth. This effect explains why keratocyte activation is observed in the cornea of patients with DE disease, along with beadings and nerve sprouts.

As mentioned above, SS implies alterations in the epithelium $(5,6,41)$. Surface morphological changes reflect the importance of the participation of epithelial cells in the inflammatory and immunologic process that characterizes keratoconjunctivitus sicca associated with SS. We found that in DE disease, the density of epithelial cells was lower compared to healthy eyes, possibly due to the enlargement of these cells during metabolic dysfunction $(34,36)$. The integrity of epithelial cells depends on the presence of intact innervation.

Another finding that proved to be in accordance with literature was the increase in Langerhans cells observed in our patients with DE disease (42). The mechanism underlying this finding is not yet fully understood, nor do we know how it links to the control of Langerhans cell migration and maturation in corneal epithelium. It was assumed that their migration and maturation are activated in response to any type of corneal irritation and pro-inflammatory stimulations $(43,44)$. Langerhans cells, serving as professional antigenpresenting cells, plays an important role in the immune surveillance of the eye (45). The higher density and more mature status of Langerhans cells in the central corneal epithelium of patients with aqueous tear deficiency may result from the inward migration of resident Langerhans cells at the limbus and peripheral cornea; it is likely they were rendered into a more mature phenotype by pro-inflammatory cytokines or chemokines.

Finally, another finding was that the thickness of the cornea was significantly reduced in our patients with DE disease, as in previous literature $(6,7,40,46-48)$. These changes occur for keratoconjunctivitus sicca in general (47) and for keratoconjunctivitus sicca combined with autoimmune disease $(5,36)$. One possible hypothesis for this thinning is that it is caused by increased apoptotic and proteolytic phenomena of the stroma. However, other authors consider it to potentially be the result of increased tangential forces that act on the epithelial surface, which can be abnormally irregular (16).

Therefore, the study was able to highlight the morphological IVCM corneal alterations in our patients and to show the efficacy of AS eye drops in symptom reduction. Nonetheless the most relevant data of this study are the changes of the ocular surface after AS therapy, data obtained with IVCM. In fact, we found that despite equally severe clinical signs (Schirmer's test, TBUT, corneal staining and thickness results), the symptoms were significantly better in patients treated with AS eye drops than in patients treated with AT; in literature, this effect of AS eye drops on the symptoms has been already described, but only a few studies have investigated the effect on the cornea of this therapy, and to our knowledge, no one has studied these changes using 
IVCM. The use of this technique allowed us to analyze the response of corneal nerves and corneal cells after therapy with AS eye drops. We can say that in patients undergoing AS eye drop therapy, the diversity of symptoms was accompanied by no change in clinical signs (Schirmer's test, TBUT, corneal staining and thicknesses), but there were some noticeable changes in structural IVCM data of the cornea, such as the number of bead-like formations and the number of sub-basal nerve branches.

One limitation of this study was that we did not take into account the degree of the severity of DE disease before the beginning of the therapy.

In conclusion, patients treated with AS eye drops, with the same results for Schirmer's test, TBUT, corneal staining and thicknesses, have a lower OSDI than patients in AT therapy, associated with the better innervation in particular evidenced by fewer bead-like formations, and fewer sub-basal nerve branches. The major contribution to trophism given by a AS eye drops may explain this, as it leads to a reduction of neurotrophic disease. Matsumoto et al. showed that therapy with AS eye drops, thanks to nerve growth factor and surfactant proteins, can help in healing neurotropic keratopathy by facilitating nerve healing and epithelialization (49). This is also supported by investigations carried out by Lopez-Garcia et al. (50).

We justify the unchanged data of the Schirmer's test and TBUT with the belief that AS eye drops are not able to act persistently on the quantity and constitution of tears. We explain the unchanged morphology of epithelium, and corneal vital staining with the idea that the ocular surface could be considered in an initial phase of compensation, that starts with changes of the sub-basal nerve plexus. With this belief, we can speculate that the persistent use of AS eye drops could lead to progressive improvement in corneal morphology and eventually in clinical signs. With this aim, the observation time should be extended to over one year.

\section{References}

1 No author listed: Research in dry eye: report of the Research Subcommittee of the International Dry Eye WorkShop. Ocul Surf 5: 179-193, 2007.

2 Benitez-Del-Castillo JM, Acosta MC, Wassfi MA, Díaz-Valle D, Gegúndez JA, Fernandez C and García-Sánchez J: Relation between corneal innervation with confocal microscopy and corneal sensitivity with noncontact esthesiometry in patients with dry eye. Invest Ophthalmol Vis Sci 48: 173-181, 2007.

3 Patel DV and McGhee $\mathrm{CN}$ : In vivo confocal microscopy of human corneal nerves in health, in ocular and systemic disease, and following corneal surgery: a review. Br J Ophthalmol 93: 853-860, 2009.

4 Benitez del Castillo JM, Wasfy MA, Fernandez C and GarciaSanchez J: An in vivo confocal masked study on corneal epithelium and sub-basal nerves in patients with dry eye. Invest Ophthalmol Vis Sci 45: 3030-3035, 2004.
5 Villani E, Galimberti D, Viola F, Mapelli C and Ratiglia R: The cornea in Sjogren's syndrome: an in vivo confocal study. Invest Ophthalmol Vis Sci 48: 2017-2022, 2007.

6 Villani E, Galimberti D, Viola F, Mapelli C, Del Papa N and Ratiglia R: Corneal involvement in rheumatoid arthritis: an in vivo confocal study. Invest Ophthalmol Vis Sci 49: 560-564, 2008.

7 Erdelyi B, Kraak R, Zhivov A, Guthoff R and Németh J: In vivo confocal laser scanning microscopy of the cornea in dry eye. Graefes Arch Clin Exp Ophthalmol 245: 39-44, 2007.

8 Ralph RA, Doane MG and Dohlman CH: Clinical experience with a mobile ocular perfusion pump. Arch Ophthalmol 93: 1039-1043, 1975.

9 Jeng BH: Use of autologous serum in the treatment of ocular surface disorders. Arch Ophthalmol 129: 1610-1612, 2011.

10 Tsubota K, Goto E, Shimmura S and Shimazaki J: Treatment of persistent corneal epithelial defect by autologous serum application. Ophthalmology 106: 1984-9, 1999.

11 Poon AC, Geerling G, Dart JK, Fraenkel GE and Daniels JT: Autologous serum eyedrops for dry eyes and epithelial defects: clinical and in vitro toxicity studies. Br J Ophthalmol 85: 1188$1197,2001$.

12 Young AL, Cheng AC, Ng HK, Cheng LL, Leung GY and Lam DS: The use of autologous serum tears in persistent corneal epithelial defects. Eye 18: 609-614, 2004.

13 Schrader S, Wedel T, Moll R and Geerling G: Combination of serum eye drops with hydrogel bandage contact lenses in the treatment of persistent epithelial defects. Graefes Arch Clin Exp Ophthalmol 244: 1345-1349, 2006.

14 Jeng BH and Dupps WJ, Jr: Autologous serum 50\% eyedrops in the treatment of persistent corneal epithelial defects. Cornea 28: 1104-1108, 2009.

15 Choi JA and Chung SH: Combined application of autologous serum eye drops and silicone hydrogel lenses for the treatment of persistent epithelial defects. Eye Contact Lens 37: 370-373, 2011.

16 Noble BA, Loh RS, MacLennan S, Pesudovs K, Reynolds A, Bridges LR, Burr J, Stewart O and Quereshi S: Comparison of autologous serum eye drops with conventional therapy in a randomised controlled crossover trial for ocular surface disease. Br J Ophthalmol 88: 647-652, 2004.

17 Tsubota K, Goto E, Fujita H, Ono M, Inoue H, Saito I and Shimmura S: Treatment of dry eye by autologous serum application in Sjogren's syndrome. Br J Ophthalmol 83: 390-395, 1999.

18 Rocha EM, Pelegrino FS, de Paiva CS, Vigorito AC and de Souza CA: GVHD dry eyes treated with autologous serum tears. Bone Marrow Transplant 25: 1101-1103, 2000.

19 Tananuvat N, Daniell M, Sullivan LJ, Yi Q, McKelvie P, McCarty DJ and Taylor HR: Controlled study of the use of autologous serum in dry eye patients. Cornea 20: 802-806, 2001.

20 Takamura E, Shinozaki K, Hata H, Yukari J and Hori S: Efficacy of autologous serum treatment in patients with severe dry eye. Adv Exp Med Biol 506: 1247-1250, 2002.

21 Ogawa Y, Okamoto S, Mori T, Yamada M, Mashima Y, Watanabe R, Kuwana M, Tsubota K, Ikeda Y and Oguchi Y: Autologous serum eye drops for the treatment of severe dry eye in patients with chronic graft-versus-host disease. Bone Marrow Transplant 31: 579-583, 2003.

22 Kojima T, Ishida R, Dogru M, Goto E, Matsumoto Y, Kaido M and Tsubota $\mathrm{K}$ : The effect of autologous serum eyedrops in the treatment of severe dry eye disease: a prospective randomized case-control study. Am J Ophthalmol 139: 242-246, 2005. 
23 Leite SC, de Castro RS, Alves M, Cunha DA, Correa ME, da Silveira LA, Vigorito AC, de Souza CA and Rocha EM: Risk factors and characteristics of ocular complications, and efficacy of autologous serum tears after haematopoietic progenitor cell transplantation. Bone Marrow Transplant 38: 223-227, 2006.

24 Villani E, Magnani F, Viola F, Santaniello A, Scorza R, Nucci P and Ratiglia R: In vivo confocal evaluation of the ocular surface morpho-functional unit in dry eye. Optom Vis Sci 90: 576-586, 2013.

25 Semeraro F, Forbice E, Braga O, Bova A, Di Salvatore A and Azzolini C: Evaluation of the efficacy of $50 \%$ autologous serum eye drops in different ocular surface pathologies. BioMed Res Int 826970, 2014.

26 Cavanagh HD, Petroll WM, Alizadeh H, He YG, McCulley JP and Jester JV. Clinical and diagnostic use of in vivo confocal microscopy in patients with corneal disease. Ophthalmology 100: 1444-1454, 1993.

27 Lopez JD, del Castillo JM, Lopez CD and Sanchez JG: Confocal microscopy in ocular chrysiasis. Cornea 22: 573-575, 2003.

28 Schein OD, Tielsch JM, Munoz B, Bandeen-Roche K and West $\mathrm{S}$ : Relation between signs and symptoms of dry eye in the elderly. A population-based perspective. Ophthalmology 104: 1395-1401, 1997.

29 Hay EM, Thomas E, Pal B, Hajeer A, Chambers H and Silman AJ: Weak association between subjective symptoms and/or bjective testing for dry eyes and dry mouth: results from a population-based study. Ann Rheum Dis 57: 20-24, 1998.

30 Nichols KK, Nichols JJ and Mitchell GL: The lack of association between signs and symptoms in patients with dry eye disease. Cornea 23: 762-770, 2004.

31 Bjerrum KB: Test and symptoms in keratoconjunctivitis sicca and their correlation. Acta Ophthalmol Scand 74: 436-441, 1996.

32 Adatia FA, Michaeli-Cohen A, Naor J, Caffery B, Bookman A and Slomovic A: Correlation between corneal sensitivity, subjective dry eye symptoms and corneal staining in Sjogren's syndrome. Can J Ophthalmol 39: 767-771, 2004.

33 Labbe A, Alalwani H, Van Went C, Brasnu E, Georgescu D and Baudouin $\mathrm{C}$ : The relationship between sub-basal nerve morphology and corneal sensation in ocular surface disease. Invest Ophthalmol Vis Sci 53: 4926-4931, 2012.

34 Benitez del Castillo JM, Wasfy MA, Fernandez C and GarciaSanchez J: An in vivo confocal masked study on corneal epithelium and sub-basal nerves in patients with dry eye. Invest Ophthalmol Vis Sci 45: 3030-3035, 2004.

35 Hosal BM, Ornek N, Zilelioglu G and Elhan AH: Morphology of corneal nerves and corneal sensation in dry eye: a preliminary study. Eye 19: 1276-1279, 2005.

36 Tuominen IS, Konttinen YT, Vesaluoma MH, Moilanen JA, Helintö M and Tervo TM: Corneal innervation and morphology in primary Sjogren's syndrome. Invest Ophthalmol Vis Sci 44: 2545-2549, 2003.
37 Zhang M, Chen J, Luo L, Xiao Q, Sun M and Liu Z: Altered corneal nerves in aqueous tear deficiency viewed by in vivo confocal microscopy. Cornea 24: 818-824, 2005.

38 Schiffman RM, Christianson MD, Jacobsen G, Hirsch JD and Reis BL: Reliability and validity of the Ocular Surface Disease Index. Arch Ophthalmol 118: 615-621, 2000.

39 Murphy CJ, Marfurt CF, McDermott A, Bentley E, Abrams GA, Reid TW and Campbell S: Spontaneous chronic corneal epithelial defects (SCCED) in dogs: clinical features, innervation, and effect of topical SP, with or without IGF-1. Invest Ophthalmol Vis Sci 42: 2252-2261, 2001.

40 Harrison DA, Joos C and Ambrosio Jr R: Morphology of corneal basal epithelial cells by in vivo slit-scanning confocal microscopy. Cornea 22: 246-248, 2003.

41 Patel S, McLaren J, Hodge D and Bourne W: Normal human keratocyte density and corneal thickness measurement by using confocal microscopy in vivo. Invest Ophthalmol Vis Sci 42: 333339, 2001.

42 Lin H, Li W, Dong N, Chen W, Liu J, Chen L, Yuan H, Geng Z and Liu Z: Changes in corneal epithelial layer inflammatory cells in aqueous tear-deficient dry eye. Invest Ophthalmol Vis Sci 51: 122-128, 2010.

43 Zhivov A, Stave J, Vollmar B and Guthoff R: In vivo confocal microscopic evaluation of Langerhans cell density and distribution in the corneal epithelium of healthy volunteers and contact lens wearers. Cornea 26: 47-54, 2007.

44 Dekaris I, Zhu SN and Dana MR: TNF-alpha regulates corneal Langerhans cell migration. J Immunol 162: 4235-4239, 1999.

45 Gillette TE, Chandler JW and Greiner JV: Langerhans cells of the ocular surface. Ophthalmology 89: 700-711, 1982.

46 Liu Z and Pflugfelder SC: Corneal thickness is reduced in dry eye. Cornea 18: 403-407, 1999.

47 Li HF, Petroll WM, Moller-Pedersen T Maurer JK, Cavanagh HD and Jester JV: Epithelial and corneal thickness measurements by in vivo confocal microscopy through focusing (CMTF). Curr Eye Res 16: 214-221, 1997.

48 McLaren JW, Nau CB, Erie JC and Bourne WM: Corneal thickness measurement by confocal microscopy, ultrasound, and scanning slit methods. Am J Ophthalmol 137: 1011-1020, 2004.

49 Matsumoto Y, Dogru M, Goto E, Ohashi Y, Kojima T, Ishida R and Tsubota K: Autologous serum application in the treatment of neurotrophic keratopathy. Ophthalmology 111: 1115-1120, 2004.

50 Lopez-Garcia JS, Rivas L, Garcia-Lozano I and Murube J: Autologous serum eyedrops in the treatment of aniridic keratopathy. Ophthalmology 115: 262-267, 2008.

Received July 6, 2016

Revised July 26, 2016

Accepted August 2, 2016 